https://doi.org/10.15407/ujpe66.1.87

\title{
ANATOLY GLIBOVYCH ZAGORODNY (to the 70th anniversary of his birth)
}

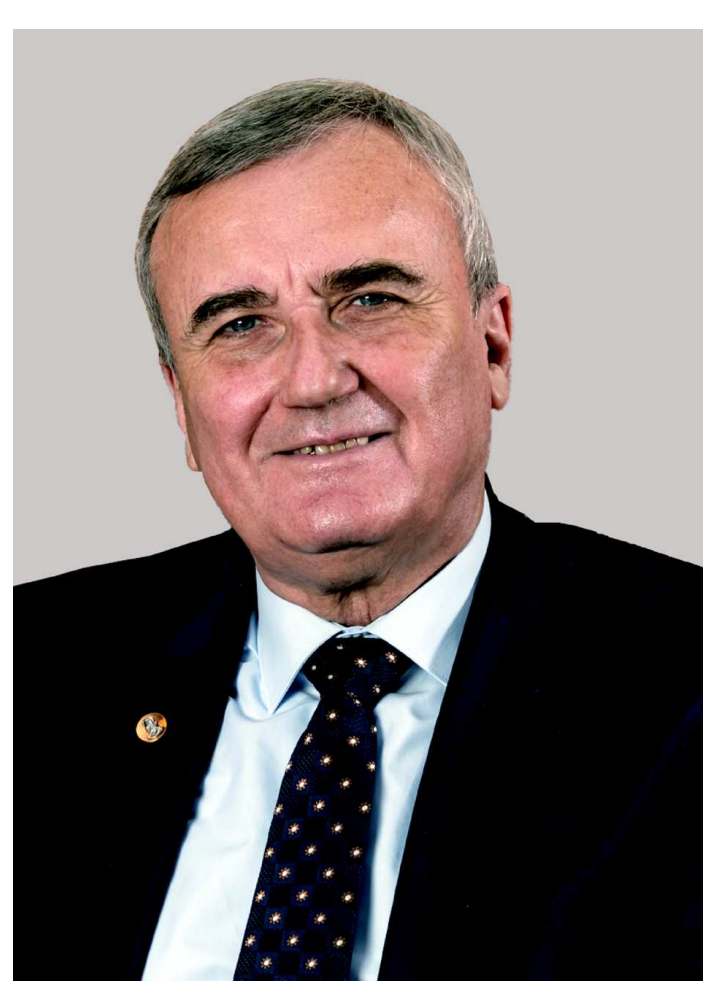

January 2021 marks the 70th birthday of Anatoly Glibovych Zagorodny, a renowned theoretical physicist and the President of the National Academy of Sciences of Ukraine (NASU).

Anatoly Glibovych was born on January 29, 1951 in the village of Velyka Bagachka in the Poltava region in the family of a doctor. There, he spent his childhood and school years. He has got a higher education at the Faculty of Radiophysics: first at the Kharkiv Institute of Radio Electronics and then at the Kharkiv University. After graduating from the University in 1972, he moved to Kyiv and became a research intern ISSN 2071-0194. Ukr. J. Phys. 2021. Vol. 66, No. 1 at the Bogolyubov Institute for Theoretical Physics (ITP) of the NASU. All further scientific and labor activity of A.G. Zagorodny was connected with the Institute and the NASU.

When working at the Institute, he combined his scientific work with the administrative activity, sequentially holding the positions of the Scientific secretary, the Head of the department, and the Deputy Director for scientific work. In 2003, A.G. Zagorodny became the Director of the Institute. Since 2009, he had also been engaging in the organization of scientific activities at the Academy level as the Chief Scientific Secretary (in 2009-2011) and the Vice President (in 2011-2020). In October 2020, the General Meeting of the NASU elected A.G. Zagorodny the President of the National Academy of Sciences of Ukraine.

The propensity of Anatoly Zagorodny to theoretical research arose during his student years at the University. When being a senior student, he got interested in plasma physics. It was the time of the rapid development in plasma researches when a competition aimed at reaching the record parameters for the plasma confinement in magnetic traps was in full swing. The variety of plasma systems, as well as the complicated character of the processes taking place in them, opened a wide field of activity for theorists. During his internship and postgraduate studies, Anatoly Zagorodny persistently mastered the methods of statistical theory that are based on the works by M.M. Bogolyubov and studied the theory of fluctuations in plasma on the basis of the works by O.G. Sitenko. The application of the consistent statistical description became a key to solution of a wide scope of problems that A.G. Zagorodny would face in the future.

His first original research, which was carried out under the supervision of I.P. Yakimenko, concerned 
the influence of the plasma system boundary on the spectrum and magnitude of fluctuations. Plasma was considered as a nonequilibrium system. The confinement and the nonequilibrium character are inherent to most plasma systems under laboratory conditions. The corresponding works performed by A.G. Zagorodny were important, in particular, for the improvement of plasma diagnostic methods that are based on the analysis of radiation scattered by fluctuations. There appeared his publications in such journals as Physica Scripta, Fizika Plazmy (Plasma Physics Reports), Ukrainian Journal of Physics, and Journal of Plasma Physics, as well as in numerous ITP preprints. The latter, at that time, were almost the only mean of communication with the world centers of physical research. Those works formed the basis of the candidate's dissertation defended by A.G. Zagorodny in 1978.

The next cycle of works by A.G. Zagorodny was motivated by the observation of a giant resonance in plasma-molecular systems. Anatoly Glibovych continued the study of electromagnetic fluctuations in confined, but more complicated plasma systems with a molecular component. In the co-authorship with I.P. Yakimenko and Yu.L. Klimontovich, a substantial contribution to the theory of such media was made. Kinetic equations with the collision integral of the Balescu-Lenard type were obtained for free and bound charged particles, and the influence of the plasma boundary on the spatial distributions of electrons, ions, and molecules was studied. A.G. Zagorodny developed the bremsstrahlung theory that involves the mutual scattering of plasma particles and molecules, their ionization and recombination, and the scattering of particles by collective fluctuations. As a result, a number of new effects in plasma with the molecular component were predicted. On the basis of the corresponding publications, which include the monograph "The Statistical Theory of PlasmaMolecular Systems", the doctoral dissertation was defended in 1990.

It is worth noting that the Institute for Theoretical Physics, which was created in 1966, was planned to be a research center with broad international links. In 1971 and 1974, the Institute hosted two large international conferences on plasma theory, which were organized by O.G. Sitenko. Anatoly Zagorodny eagerly joined this activity. His first scientific contacts were maintained in the future and affected the direction of his further research. In the 1990s, A.G. Zagorodny got interested in the spectrum of electromagnetic waves scattered by plasma fluctuations, which were registered in one of French laboratories. Their nonGaussian form could not be explained in the framework of the classical theory of fluctuations. A.G. Zagorodny together with O.G. Sitenko developed the theory of turbulent plasma with diffusion-drift fluid motion. This model allowed them to describe the unusual features of fluctuations and the corresponding scattering spectra.

Later, the interest in turbulent plasma was extended to anomalous transport phenomena unexplainable in terms of Markovian processes. Due to nonequilibrium - this is a usual state of plasma under laboratory or outer space conditions - intense eigen oscillations can be excited in plasma. The anomalous transport process caused by the scattering of particles by collective excitations dominates over the classical diffusion associated with pair collisions. The knowledge about the transport process character and the calculation of corresponding transport coefficients are necessary in order to estimate the time of the plasma confinement in laboratory devices. A.G. Zagorodny formulated kinetic equations for systems with nonMarkovian relaxation processes and found their solutions, which explained why different conditions give rise to the appearance of super- or subdiffusion regimes in plasma.

The next object of research became plasma with solid impurities (dust particles). Its study began to develop very rapidly soon after the periodic patterns formed from dust particles in a plasma environment had been observed for the first time. At the very beginning, such plasma was considered as an exotic object. The community of physicists dealing with controlled nuclear fusion was somewhat skeptical about the relevance of the report made by A.G. Zagorodny at the international conference in 1996. But the time had passed, and there arose a separate direction of researches in which the method of describing the dusty plasma is applied to study the behavior of impurities in thermonuclear devices.

The specific feature of the dust component in comparison with the molecular one consists in that the charge of dust particles exceeds the elementary one by four or even more orders of magnitude. Moreover, it does not remain constant. Dust particles are much more massive than ions or molecules. At the same 
time, they are not macroscopic objects, because their behavior is governed by microfields in their environment. They absorb or emit electrons, capture them onto closed orbits, so that their interaction with the plasma environment is rather complicated. A.G. Zagorodny formulated rigorous microscopic equations for plasma with dust impurities and derived a chain of Bogolyubov equations for them. For dust particles, the effective interaction potentials which are responsible for the formation of patterns from those particles at various temperatures were found. It was predicted that if the conditions for the drift-diffusion approximation are satisfied, the long-range potentials and the effect of negative friction arise. They are unordinary phenomena resulting from the influence of plasma flows on dust particles. The theory of electromagnetic fluctuations in dusty plasma was developed taking the charge fluctuations of dust particles into account. Those results are important for plasma diagnostics.

In recent years, A.G. Zagorodny pays attention to the establishment of relationships for the energy characteristics of fluctuation fields in a medium with the temporal and spatial dispersion beyond the spectral interval of medium transparency (this is a classical problem that does not have a rigorous solution till now), and to developing a statistical description for a wide class of many particle systems that include the so-called complex plasma.

This very short list of Anatoly Glibovych's works does not include a large body of his publications on other topics. Their total number exceeds 200. He regularly reported the results of his research at prestigious international congresses on plasma physics as an invited lecturer. A.G. Zagorodny was also a member of the organizing committees at a number of international conferences. In 2004-2006, he was the chairman of the International Congress on Plasma Physics. For his scientific achievements, he was awarded the title of Laureate of the State Prize of Ukraine in Science and Technology (2005), the Honored Worker of Science and Technology of Ukraine (2012), and the laureate of the Sinel'nikov (1991), Bogolyubov (2012), and Davydov (2019) prizes of the NASU.

In 1997, A.G. Zagorodny was elected a Corresponding Member and, in 2006, an Academician of the NASU. In 2015, he became a member of the Shevchenko Scientific Society. Besides that, A.G. Zagorod- ny is a foreign member of the Russian Academy of Sciences (2011); a foreign corresponding member of the Austrian Academy of Sciences (2012); a corresponding member of the International Academy of Sciences, Humanities, and Arts (Paris, 2004); the Honorary Professor of the Jilin University (2017) and the laureate of the Qilu Friendship Award of the People's Government of Shandong Province (China, 2019); Honoris Causa of the V.N. Karazin National University of Kharkiv (2010), the I.I. Mechnikov National University of Odesa (2015), the Taras Shevchenko National University of Kyiv (2017), and the Institute of Condensed Matter Physics of the NASU (2008); and the Honorary Scientist of the International Institute of Applied Systems Analysis (2020).

When communicating with Anatoly Glibovych, people more often face only one of the directions of his activity. Along with a huge amount of scientificorganizational, scientific-coordinational, and expert work, he continues to pay attention to science even in the busiest time periods; then, mostly weekends and holidays are used for this purpose. Till his recent election as the President of the NASU, he coordinated the scientific activity of the Academy as the Vice President. He is the Director of the Bogolyubov Institute for Theoretical Physics, the chairman of its council for the defense of dissertations, the supervisor of target programs, the head of some scientific councils of the NASU, the supervisor of the programs of international cooperation between the NASU and the European Union, the chairman of the commission on the analysis of the scientific heritage of Academician V.I. Vernadsky and the comission on the work with scientific youth, as well as the Editorin-Chief of the Ukrainian Journal of Physics, with this list being incomplete. He is also a member of the Committee on the State Prizes of Ukraine and the Committee for Awarding the Prizes of the Cabinet of Ministers, the chairman of the supervisory board of the V.N. Karazin National University of Kharkiv, a deputy chairman of the National Commission of Ukraine for UNESCO, a scientific delegate of Ukraine to the CERN Council, the chairman of the National Committee of UNESCO's Man and the Biosphere Programme, a member of the Scientific Advisory Committee in a number of the International Conferences on Dusty Plasma Physics, a representative of Ukraine in the Council of representatives and participants at the European Commission Joint Re- 
search Centre, a council member at the IIASA, and a member of the Ukraine-Euratom Research and Innovation Committee.

A.G. Zagorodny pays the large attention to the training of young scientists not only as the chairman of the commission on the work with scientific youth, but also as a lecturer. Since 2018, he has been heading the Department of Theoretical and Mathematical Physics at the Kyiv Academic University. For decades, he lectured special courses on plasma physics at the Taras Shevchenko National University of Kyiv and was a professor at the Kyiv-Mohyla Academy. Anatoly Glibovych is an author and a co-author of several monographs and textbooks. He was a supervisor of two doctoral and five Ph.D. dissertations.

For his scientific and scientific-managerial work, A.G. Zagorodny was awarded the Order of Merit of the third (2008) and second (2016) degrees, and the Diploma of the Supreme Council of Ukraine (2004).

The friends and colleagues sincerely congratulate Anatoly Glibovych on his jubilee, wish him the implementation of his plans and the relevant support from the scientific community, as well as new creative achievements and sound health.

O.S. BAKAI, V.P. GUSYNIN, V.I. ZASENKO, G.M. ZINOVJEV, YU.I. IZOTOV, B.I. LEV, V.M. LOKTEV, I.M. MRYGLOD, E.G. PETROV, YU.O. SITENKO, YU.V. SLYUSARENKO, M.F. SHULGA, I.R. YUKHNOVSKII

The Editorial Board and the editorial staff of the Ukrainian Physical Journal also congratulate our dear Anatoly Glibovych, the Editor-in-Chief of UJP, and wish him the good health and great successes in all his endeavours! 